TRANSACTIONS OF THE

AMERICAN MATHEMATICAL SOCIETY

Volume 351, Number 9, Pages 3843-3855

S 0002-9947(99)02316-8

Article electronically published on May 21, 1999

\title{
DIRECT SUM DECOMPOSITIONS OF INFINITELY GENERATED MODULES
}

\author{
D. J. BENSON AND WAYNE W. WHEELER
}

\begin{abstract}
Almost all of the basic theorems in the representation theory of finite groups have proofs that depend upon the Krull-Schmidt Theorem. Because this theorem holds only for finite-dimensional modules, however, the recent interest in infinitely generated modules raises the question of which results may hold more generally. In this paper we present an example showing that Green's Indecomposability Theorem fails for infinitely generated modules. By developing and applying some general properties of idempotent modules, we are also able to construct explicit examples of modules for which the cancellation property fails.
\end{abstract}

\section{INTRODUCTION}

The representation theory of finite groups has traditionally concentrated on finitely generated modules over the group algebra. Recent work $[1,3,4,5]$ has made it clear, however, that infinitely generated modules play an important role even if one's real goal is a better understanding of finitely generated modules. For this reason it should be useful to try to extend known results in representation theory by omitting the finiteness conditions on the modules. One of the main objectives of this paper is to study the analogue of Green's Indecomposability Theorem in this greater generality.

Suppose that $G$ is a finite group and $k$ is a field of characteristic $p>0$. Green's Indecomposability Theorem [10] states that if $H$ is a normal subgroup of index $p$ in $G$ and $M$ is a finitely generated absolutely indecomposable $k H$-module, then the induced module $M^{\top}{ }^{G}$ is also absolutely indecomposable. The usual proof of this result depends upon the Krull-Schmidt Theorem. Work of Brenner and Ringel [7] shows, however, that for infinitely generated modules the Krull-Schmidt Theorem fails spectacularly whenever $G$ has noncyclic Sylow $p$-subgroups. In Section 2 we present a counterexample showing that Green's result also fails without the assumption that $M$ is finitely generated.

Sections 3 and 4 are devoted to studying the failure of the Krull-Schmidt property in somewhat more detail. This work begins by considering endomorphism rings of certain types of infinitely generated modules. The arguments of Section 4 then show, in effect, that every complete intersection is isomorphic to $\operatorname{End}_{k G}(M) / I$ for some elementary abelian $p$-group $G$, some countably generated module $M$, and some nilpotent ideal $I$ of $\operatorname{End}_{k G}(M)$. If $n \geq 5$ and $G$ is elementary abelian of rank

Received by the editors November 12, 1997.

1991 Mathematics Subject Classification. Primary 20C20.

Both authors are partially supported by the NSF. 
$2 n+1$, then Raynaud's examples of modules that are stably free but not free [12] allow us to exhibit modules $M$ and $N$ such that $M$ is indecomposable and

$$
M^{n} \cong M \oplus N
$$

but $M^{n-1} \neq N$. Such examples also exist for certain smaller values of $n$. If $k G$ has infinite representation type, then the work of Brenner and Ringel proves the existence of modules for which the cancellation property fails in this way. Their methods leave it far from obvious what such modules might look like, however, and we believe that it is a valuable exercise to construct explicit examples as we have done.

\section{Green's Indecomposability Theorem}

The goal of this section is to produce an example showing that the statement of Green's Indecomposability Theorem fails for infinitely generated modules. The example is based upon Rickard's construction of idempotent modules in [13]. We begin with a brief discussion of the necessary definitions and preliminary results.

Let $G$ be a finite group, and let $k$ be an algebraically closed field of characteristic $p>0$. Recall that the objects of the stable category $k G$-Mod consist of all left $k G$ modules, and the morphisms are equivalence classes of $k G$-homomorphisms; two homomorphisms $f: M \rightarrow N$ and $g: M \rightarrow N$ are equivalent if $f-g$ factors through a projective module. The collection of morphisms from $M$ to $N$ in $k G$ Mod is denoted by $\underline{\operatorname{Hom}}_{k G}(M, N)$. Because $k G$ is a self-injective algebra, $k G$-Mod is a triangulated category (Theorem I.2.6 of [11]), and the translation functor is given by $\Omega^{-1}$. The full subcategory of $k G$-Mod consisting of all finitely generated $k G$-modules is denoted by $k G$-mod.

A triangulated subcategory $\mathcal{C}$ of $k G$-mod is thick if it is closed under taking direct summands of objects. If $\mathcal{C}$ is a thick subcategory of $k G$-mod such that $M \otimes N$ is in $\mathcal{C}$ whenever $M$ is in $\mathcal{C}$ and $N$ is in $k G$-mod, then we say that $\mathcal{C}$ is an ideal subcategory of $k G$-mod. Let $\mathcal{C}^{\oplus}$ denote the smallest full triangulated subcategory of $k G$-Mod that contains $\mathcal{C}$ and is closed under arbitrary direct sums, and write $\mathcal{C}^{\perp}$ for the subcategory of $k G$-Mod consisting of all modules $M$ such that $\underline{\operatorname{Hom}}_{k G}(C, M)=0$ for all $C \in \mathcal{C}$. The objects of $\mathcal{C}^{\perp}$ are said to be $\mathcal{C}$-local. With this notation and terminology we can now state the following result of Rickard [13].

Proposition 2.1. Let $\mathcal{C}$ be an ideal subcategory of $k G$-mod. For any object $M$ in $k G$-Mod there is a triangle

$$
E_{\mathcal{C}}(M) \rightarrow M \rightarrow F_{\mathcal{C}}(M) \rightarrow \Omega^{-1}\left(E_{\mathcal{C}}(M)\right)
$$

such that $E_{\mathcal{C}}(M)$ is in $\mathcal{C}^{\oplus}$ and $F_{\mathcal{C}}(M)$ is in $\mathcal{C}^{\perp}$, and such a triangle is unique up to isomorphism. The morphism $E_{\mathcal{C}}(M) \rightarrow M$ is the universal map in $k G$ Mod from an object of $\mathcal{C}^{\oplus}$ to $M$, and $M \rightarrow F_{\mathcal{C}}(M)$ is the universal map from $M$ to an object of $\mathcal{C}^{\perp}$. The modules $E_{\mathcal{C}}(k)$ and $F_{\mathcal{C}}(k)$ are idempotent in the sense that $E_{\mathcal{C}}(k) \otimes E_{\mathcal{C}}(k) \cong E_{\mathcal{C}}(k)$ and $F_{\mathcal{C}}(k) \otimes F_{\mathcal{C}}(k) \cong F_{\mathcal{C}}(k)$ in $k G$-Mod. Moreover, $M \otimes E_{\mathcal{C}}(k) \cong E_{\mathcal{C}}(M)$ and $M \otimes F_{\mathcal{C}}(k) \cong F_{\mathcal{C}}(M)$ in $k G$-Mod for any module $M$.

For the remainder of this section $k$ denotes an algebraically closed field of characteristic two. Let $H$ be an elementary abelian group of order four generated by commuting involutions $h_{1}$ and $h_{2}$, and let $G$ be the group generated by $H$ and an involution $g$ satisfying $g h_{1}=h_{1} h_{2} g$ and $g h_{2}=h_{2} g$. Then $H$ is a normal subgroup of $G$ of index two, and $G$ is isomorphic to the dihedral group $D_{8}$. 
Set $X_{i}=h_{i}-1 \in k H$ for $i=1,2$. If we identify $H^{1}(H, k)$ with the vector space $\operatorname{Hom}(H, k)$ of group homomorphisms into $k$, then there are elements $\zeta_{1}, \zeta_{2} \in H^{1}(H, k)$ satisfying $\zeta_{1}\left(h_{1}\right)=\zeta_{2}\left(h_{2}\right)=1$ and $\zeta_{1}\left(h_{2}\right)=\zeta_{2}\left(h_{1}\right)=0$, and $H^{*}(H, k)$ is the polynomial ring generated by $\zeta_{1}$ and $\zeta_{2}$. Let $\mathcal{C}$ be the subcategory of $k H$-mod consisting of all finitely generated $k H$-modules $N$ whose variety $V_{H}(N)$ is contained in $V_{H}\left(\zeta_{2}\right)$, and let $M=F_{\mathcal{C}}(k)$ be the corresponding idempotent $\mathrm{kH}$-module. More explicitly, $M$ can be described as the module with generators $x_{0}, x_{1}, x_{2}, \ldots$ and relations

$$
X_{1} x_{i}+X_{2} x_{i+1}=0 \text { for all } i \geq 0 .
$$

This module has a unique $k H$-endomorphism $\alpha$ satisfying $\alpha\left(x_{i}\right)=x_{i+1}$. The relations (2.1) can be rewritten in terms of the endomorphism $\alpha$ as

$$
\left(X_{1}+X_{2} \alpha\right) m=0 \text { for all } m \in M \text {. }
$$

Using Proposition 7.3 of [9] and the techniques of [8], one can show that $\underline{\operatorname{End}}_{k H}(M)$ $=k[\alpha]$. This fact is also easy to prove directly.

If the commutative ring $k H[\alpha]$ is regarded as a free left $k H$-module, then there is a $k H$-homomorphism onto $M$ sending $\alpha^{i}$ to $x_{i}$ for $i \geq 0$. Thus by $(2.2)$ there is an isomorphism of $k H$-modules

$$
M \cong k H[\alpha] /\left(X_{1}+X_{2} \alpha\right) .
$$

The conjugation action of $g$ on $k H$ extends to an action on the ring $k H[\alpha]$ satisfying $g^{-1} \alpha g=\alpha+1$, and it is easy to check that $g$ induces an action on the quotient in (2.3). Under the $k H$-isomorphism (2.3) this action of $g$ determines a $k$-linear map $\phi: M \rightarrow M$ satisfying

$$
\phi(x m)=\left(g^{-1} x g\right) \phi(m)
$$

for all $x \in k H$ and all $m \in M$. The map $\phi$ is given on the generators of $M$ by

$$
\phi\left(x_{i}\right)=\phi\left(\alpha^{i}\left(x_{0}\right)\right)=(\alpha+1)^{i}\left(x_{0}\right)=\sum_{j=0}^{i}\left(\begin{array}{l}
i \\
j
\end{array}\right) x_{j} .
$$

Now consider the induced module $M^{G}=M \oplus(g \otimes M)$. The map $m \mapsto g \otimes \phi(m)$ is a $k H$-isomorphism from $M$ to $g \otimes M$; its inverse is given by $g \otimes m \mapsto \phi(m)$, because $g$ is an involution so that $\phi^{2}=1_{M}$. Corresponding to this isomorphism is a $k G$-endomorphism $\psi: M \uparrow^{G} \rightarrow M \uparrow^{G}$ given by

$$
\psi\left(m_{1}, g \otimes m_{2}\right)=\left(\phi\left(m_{2}\right), g \otimes \phi\left(m_{1}\right)\right) .
$$

In addition, there is an endomorphism $a$ of $M^{G}$ given by

$$
a\left(m_{1}, g \otimes m_{2}\right)=\left(\alpha\left(m_{1}\right), g \otimes \alpha\left(m_{2}\right)\right) ;
$$

this is the map induced from the $k H$-endomorphism $\alpha$ of $M$. If the isomorphism $m \mapsto g \otimes \phi(m)$ is used to identify the $k H$-modules $M$ and $g \otimes M$, then $\operatorname{End}_{k H}\left(M \uparrow^{G} \downarrow_{H}\right)$ is isomorphic to $\operatorname{Mat}_{2}\left(\operatorname{End}_{k H}(M)\right)$. It is helpful to think of the endomorphisms $a$ and $\psi$ as matrices:

$$
a=\left(\begin{array}{cc}
\alpha & 0 \\
0 & \alpha+1
\end{array}\right), \quad \psi=\left(\begin{array}{cc}
0 & 1 \\
1 & 0
\end{array}\right) .
$$

Lemma 2.2. The endomorphism ring $\underline{\operatorname{End}}_{k G}\left(M \uparrow{ }^{G}\right)$ is isomorphic to the $k$-algebra with generators $a$ and $\psi$ and relations

$$
a \psi=\psi(a+1), \quad \psi^{2}=1 .
$$


Proof. Because $\operatorname{End}_{k G}\left(M \uparrow^{G}\right)$ is contained in $\operatorname{End}_{k H}\left(M \uparrow^{G} \downarrow_{H}\right)$, it can be regarded as a subring of $\operatorname{Mat}_{2}\left(\operatorname{End}_{k H}(M)\right)$. The composition of the isomorphisms

$$
\begin{aligned}
\operatorname{End}_{k H}(M) \oplus \operatorname{End}_{k H}(M) & \cong \operatorname{End}_{k H}(M) \oplus \operatorname{Hom}_{k H}(g \otimes M, M) \\
& \cong \operatorname{Hom}_{k H}\left(M \uparrow^{G} \downarrow_{H}, M\right) \\
& \cong \operatorname{End}_{k G}(M \uparrow G)
\end{aligned}
$$

is given by the map

$$
\left(\beta, \beta^{\prime}\right) \mapsto\left(\begin{array}{cc}
\beta & \beta^{\prime} \\
\phi \beta^{\prime} \phi & \phi \beta \phi
\end{array}\right)
$$

Moreover, $\beta$ and $\beta^{\prime}$ are projective endomorphisms of $M$ if and only if the corre-

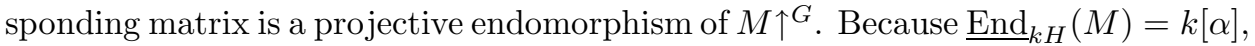
we conclude that $\operatorname{End}_{k G}\left(M \uparrow^{G}\right)$ is generated by the images of the maps $a$ and $\psi$, and the desired result follows.

The $k H$-module $M$ is indecomposable because $\operatorname{End}_{k H}(M)=k[\alpha]$, so the following result shows that Green's criterion for indecomposability fails for $M^{\top}{ }^{G}$.

Theorem 2.3. The module $M^{G}$ is decomposable.

Proof. Set $e=a(1+\psi) \in \operatorname{End}_{k G}\left(M^{G}\right)$. It is easy to check that $e$ is a nontrivial idempotent of $\operatorname{End}_{k G}\left(M \uparrow^{G}\right)$, so $M^{\top}{ }^{G}$ is decomposable.

Our next objective in this section is to study direct sum decompositions of $M \uparrow^{G}$ by considering idempotents in the endomorphism $\operatorname{ring} \operatorname{End}_{k G}\left(M \uparrow^{G}\right)$. Let $P, Q \in$ $k[x]$ be polynomials with $Q \neq 0$. Then one can check that $P(a)+Q(a) \psi$ is an idempotent in $\underline{\operatorname{End}}_{k G}\left(M \uparrow^{G}\right)$ if and only if

$$
\begin{aligned}
P(x+1) & =P(x)+1, \\
P(x) P(x+1) & =Q(x) Q(x+1) .
\end{aligned}
$$

We will describe how to find the solutions to these equations.

First consider equation (2.4a), and observe that $P(x)=x$ is a solution. Moreover, any other solution is of the form $P(x)=x+P_{0}(x)$, where $P_{0} \in k[x]$ is a polynomial satisfying

$$
P_{0}(x+1)=P_{0}(x) .
$$

Such polynomials $P_{0}$ form a subspace $V$ of the vector space $k[x]$, and the solutions of (2.4a) are the elements of the coset $x+V$. The next result describes a basis for $V$.

Proposition 2.4. Let $\mathbb{Z} / 2=\langle g\rangle$ act on $k[x]$ by $(g \cdot P)(x)=P(x+1)$ for all $P \in k[x]$. For each $n \geq 0$ there is a g-invariant polynomial of degree $2 n$ in $k[x]$, and there are no $g$-invariant polynomials of odd degree. Moreover, if $\mathcal{B}$ is a subset of $k[x]$ consisting of precisely one $g$-invariant polynomial of degree $2 n$ for all $n \geq 0$, then $\mathcal{B}$ is a basis for $V=\operatorname{soc} k[x]$.

Proof. Set $V_{-1}=0$, and for any integer $n \geq 0$ let $V_{n}$ be the $k[\mathbb{Z} / 2]$-submodule of $k[x]$ consisting of all polynomials of degree at most $n$. Then $V_{2 n+1} / V_{2 n-1}$ is a two-dimensional $k[\mathbb{Z} / 2]$-module. Because $x^{2 n+1}+(x+1)^{2 n+1}$ is a polynomial of degree $2 n$, it follows that $V_{2 n+1} / V_{2 n-1}$ is not a trivial module and must be free of rank one. It is easy to see by induction on $n$ that $V_{2 n+1}$ is free of rank $n+1$. Thus $\operatorname{soc}\left(V_{2 n+1}\right)=\operatorname{soc}\left(V_{2 n}\right)$ has dimension $n+1$ and $\operatorname{soc}\left(V_{2 n-1}\right)$ has dimension $n$, so 
$V_{2 n+1}$ has a $g$-invariant polynomial of degree $2 n$ but no $g$-invariant polynomial of degree $2 n+1$. Moreover, if $\mathcal{B}$ is a set consisting of exactly one $g$-invariant polynomial of degree $2 n$ for all $n \geq 0$, then $\mathcal{B}$ is a basis for $\operatorname{soc} k[x]$, as desired.

Now suppose that $P \in k[x]$ is a polynomial satisfying (2.4a). Write

$$
P(x) P(x+1)=\lambda\left(x+a_{1}\right)^{e_{1}} \cdots\left(x+a_{n}\right)^{e_{n}},
$$

where $a_{1}, \ldots, a_{n} \in k$ are distinct. Then for each index $i$ there is a unique $j \neq i$ such that $a_{j}=a_{i}+1$, and $e_{j}=e_{i}$. Thus $n=2 s$ for some natural number $s$. By reordering the indices if necessary, we may assume that $a_{2 i}=a_{2 i-1}+1$ for $1 \leq i \leq s$. Let $\mu \in k$ be the element satisfying $\mu^{2}=\lambda$. Then $Q \in k[x]$ satisfies $(2.4 \mathrm{~b})$ if and only if

$$
Q(x)=\mu\left(x+a_{1}\right)^{m_{1}} \cdots\left(x+a_{n}\right)^{m_{n}}
$$

for some nonnegative integers $m_{1}, \ldots, m_{n}$ such that $m_{2 i-1}+m_{2 i}=e_{2 i}$ for $1 \leq i \leq s$.

Thus we obtain a description of the polynomials satisfying (2.4). In particular, it follows that the $\operatorname{ring} \underline{\operatorname{End}}_{k G}(M \uparrow G)$ contains infinitely many nontrivial idempotents, so that the module $M \uparrow^{G}$ has infinitely many different direct sum decompositions. We end this section by showing that all of these nontrivial idempotents are actually conjugate in $\underline{\operatorname{End}}_{k G}(M \uparrow G)$.

If $\underline{\operatorname{End}}_{k G}\left(M \uparrow^{G}\right)$ is regarded as a subalgebra of $\operatorname{Mat}_{2}(k[\alpha])$ as before, then an arbitrary element $P(a)+Q(a) \psi \in \underline{\operatorname{End}}_{k G}\left(M \uparrow \uparrow^{G}\right)$ is given by the matrix

$$
\left(\begin{array}{cc}
P(\alpha) & Q(\alpha) \\
Q(\alpha+1) & P(\alpha+1)
\end{array}\right) .
$$

Thus $P(a)+Q(a) \psi$ is a unit if and only if the determinant

$$
P(\alpha) P(\alpha+1)+Q(\alpha) Q(\alpha+1)
$$

lies in $k-\{0\}$. The units of determinant one form a subgroup $U_{1}$ of the full group of units $U$ in $\underline{\operatorname{End}}_{k G}(M \uparrow G)$. If $k^{\times}$denotes the multiplicative group of the field $k$, then it is easy to see that $U=U_{1} \times k^{\times}$.

Proposition 2.5. The group $U_{1}$ of units of determinant one in $\operatorname{End}_{k G}(M \uparrow G)$ acts transitively on the collection of nontrivial idempotents in $\underline{\operatorname{End}}_{k G}(M \uparrow G)$. The stabilizer in $U_{1}$ of the idempotent $e=a(1+\psi)$ is the subgroup

$$
\operatorname{Stab}_{U_{1}}(e)=\left\{c+\left(c+c^{-1}\right) e \mid c \in k^{\times}\right\} .
$$

Proof. Let $P, Q \in k[x]$ be polynomials with $Q \neq 0$ such that $P(a)+Q(a) \psi$ is an idempotent in $\underline{\operatorname{End}}_{k G}(M \uparrow G)$, and let

$$
e_{P, Q}=\left(\begin{array}{cc}
P(\alpha) & Q(\alpha) \\
Q(\alpha+1) & P(\alpha+1)
\end{array}\right)
$$

be the corresponding idempotent matrix in $\operatorname{Mat}_{2}(k[\alpha])$. By $(2.4 \mathrm{~b})$ we can write $Q(x)=Q_{0}(x) Q_{1}(x)$, where $Q_{0}(x)$ divides $P(x)$ and $Q_{1}(x)$ divides $P(x+1)$. Because $P(x+1)=P(x)+1$, the polynomials $P(x)$ and $P(x+1)$ have no common factors, so $Q_{0}(x)$ and $Q_{1}(x)$ are unique up to scalar multiples. Moreover,

$$
P(x)=Q_{0}(x) Q_{1}(x+1) .
$$

Then one can check that

$$
u_{P, Q}=\left(\begin{array}{cc}
Q_{0}(\alpha) & Q_{1}(\alpha) \\
Q_{0}(\alpha+1) & Q_{1}(\alpha+1)
\end{array}\right)
$$


is a matrix of determinant one in $\operatorname{Mat}_{2}(k[\alpha])$ such that

$$
u_{P, Q}^{-1} e_{P, Q} u_{P, Q}=\left(\begin{array}{ll}
1 & 0 \\
0 & 0
\end{array}\right) \text {. }
$$

Now suppose that $P^{\prime}, Q^{\prime} \in k[x]$ are also polynomials with $Q^{\prime} \neq 0$ such that $P^{\prime}(a)+Q^{\prime}(a) \psi$ is an idempotent. Write $Q^{\prime}(x)=Q_{0}^{\prime}(x) Q_{1}^{\prime}(x)$ as above, and set

$$
\begin{aligned}
& \hat{Q}_{0}(x)=Q_{0}(x) Q_{1}^{\prime}(x+1)+Q_{1}(x) Q_{0}^{\prime}(x+1), \\
& \hat{Q}_{1}(x)=Q_{0}(x) Q_{1}^{\prime}(x)+Q_{1}(x) Q_{0}^{\prime}(x) .
\end{aligned}
$$

Then

$$
u_{P, Q} u_{P^{\prime}, Q^{\prime}}^{-1}=\left(\begin{array}{cc}
\hat{Q}_{0}(\alpha) & \hat{Q}_{1}(\alpha) \\
\hat{Q}_{1}(\alpha+1) & \hat{Q}_{0}(\alpha+1)
\end{array}\right)
$$

is a matrix of determinant one that conjugates $e_{P, Q}$ to $e_{P^{\prime}, Q^{\prime}}$. Moreover, the matrix $u_{P, Q} u_{P^{\prime}, Q^{\prime}}^{-1}$ corresponds to the unit $\hat{Q}_{0}(a)+\hat{Q}_{1}(a) \psi \in \underline{\operatorname{End}}_{k G}\left(M \uparrow^{G}\right)$. Thus $U_{1}$ acts transitively on the nontrivial idempotents in $\underline{\operatorname{End}}_{k G}\left(M^{\uparrow}{ }^{G}\right)$.

Finally, suppose that $P(x)=Q(x)=x$, so that $e_{P, Q}$ is the matrix corresponding to the idempotent $e=a(1+\psi)$. The stabilizer in $\mathrm{SL}_{2}(k[\alpha])$ of the matrix $e_{P, Q}$ consists of all matrices of the form

$$
u_{P, Q}\left(\begin{array}{cc}
c^{-1} & 0 \\
0 & c
\end{array}\right) u_{P, Q}^{-1}=\left(\begin{array}{cc}
c+\left(c+c^{-1}\right) \alpha & \left(c+c^{-1}\right) \alpha \\
\left(c+c^{-1}\right)(\alpha+1) & c+\left(c+c^{-1}\right)(\alpha+1)
\end{array}\right)
$$

for $c \in k^{\times}$. It follows that $\operatorname{Stab}_{U_{1}}(e)$ consists of all elements of the form

$$
c+\left(c+c^{-1}\right) a+\left(c+c^{-1}\right) a \psi=c+\left(c+c^{-1}\right) e,
$$

and this completes the proof.

Even if the coefficient field $k$ is not algebraically closed, one can define an absolutely indecomposable $k H$-module $M$ with generators $x_{0}, x_{1}, x_{2}, \ldots$ and relations given by (2.1). In this case the solutions to (2.4b) are obtained by writing

$$
P(x) P(x+1)=\lambda P_{1}(x)^{e_{1}} \cdots P_{n}(x)^{e_{n}},
$$

where $P_{1}, \ldots, P_{n}$ are distinct irreducible monic polynomials in $k[x]$ and $\lambda \in k$. The possibilities for $Q(x)$ are then determined by considering the cases $P_{i}(x)=P_{i}(x+1)$ and $P_{i}(x) \neq P_{i}(x+1)$ for each factor $P_{i}$. With this minor change all of the results proven in this section for $M$ or for $M \uparrow G$ remain valid. In particular, when $k=\mathbb{F}_{2}$, Proposition 2.5 shows that the group $U_{1}$ of units of determinant one in $\underline{\operatorname{End}}_{\mathbb{F}_{2} G}\left(M \uparrow^{G}\right)$ acts regularly on the nontrivial idempotents in $\operatorname{End}_{\mathbb{F}_{2} G}\left(M \uparrow^{G}\right)$.

\section{Graded ENDOMORPhism RINGS OF IDEMPotent MOdules}

Throughout this section $G$ denotes an arbitrary finite group, $k$ is an algebraically closed field of characteristic $p>0$, and $\mathcal{C}$ is an ideal subcategory of $k G$-mod. The objective is to study graded endomorphism rings for the idempotent module $F=F_{\mathcal{C}}(k)$ and certain related modules. The results are used in the next section to produce examples of modules for which the cancellation property fails. 
For any $k G$-modules $M$ and $N$, write $\underline{\operatorname{Hom}}_{k G}^{*}(M, N)$ for the $\mathbb{Z}$-graded vector space with

$$
\underline{\operatorname{Hom}}_{k G}^{n}(M, N)=\underline{\operatorname{Hom}}_{k G}\left(\Omega^{n} M, N\right) .
$$

Assume that $\Omega^{0}$ is the identity functor on $k G$ - $\underline{\operatorname{Mod}}$, so that $\underline{\operatorname{Hom}}_{k G}(M, N)$ is the space of elements of degree zero in $\underline{\operatorname{Hom}}_{k G}^{*}(M, N)$. If $\xi \in \underline{\operatorname{Hom}}_{k G}^{m}(M, N)$ and $\zeta \in \underline{\operatorname{Hom}}_{k G}^{n}(L, M)$, then we write $\xi \circ \zeta$ for the element of $\underline{\operatorname{Hom}}_{k G}^{m+n}(L, N)$ given by $\xi \circ \zeta=\xi\left(\Omega^{m} \zeta\right)$. For convenience we usually identify $\Omega^{n} M$ with $\Omega^{n} k \otimes M$ when no confusion is likely to arise.

Let $\eta: k \rightarrow F$ denote the universal map in $k G$-Mod from $k$ to an object of $\mathcal{C}^{\perp}$, as in Proposition 2.1. Suppose that $M$ and $N$ are in $\mathcal{C}^{\perp}$, so that $\eta \otimes 1_{M}: M \rightarrow F \otimes M$ and $\eta \otimes 1_{N}: N \rightarrow F \otimes N$ are stable isomorphisms. If $\zeta \in \operatorname{End}_{k G}^{m}(F, F)$ and $f \in \underline{\operatorname{Hom}}_{k G}^{n}(M, N)$, then there is a unique map $\zeta \cdot f: \Omega^{m+n} M \rightarrow N$ such that the following diagram commutes:

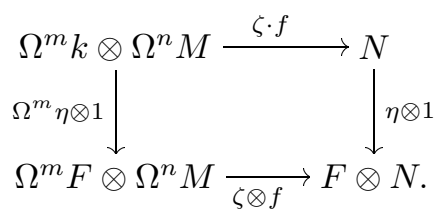

One can check that this definition makes $\underline{\operatorname{Hom}}_{k G}^{*}(M, N)$ into a graded left module for $\operatorname{End}_{k G}^{*}(F)$. It is easy to see that

$$
\zeta \cdot f=\left(\zeta \cdot 1_{N}\right) \circ\left(1_{\Omega^{m} k} \otimes f\right)=f \circ\left(\zeta \cdot 1_{\Omega^{n} M}\right) .
$$

Similarly, $\underline{\operatorname{Hom}}_{k G}^{*}(M, N)$ has a right module structure given by setting $f \cdot \zeta=$ $f \circ\left(\zeta \cdot 1_{M}\right)$, and this definition makes $\underline{\operatorname{Hom}}_{k G}^{*}(M, N)$ into an $\underline{\operatorname{End}}_{k G}^{*}(F)$-bimodule. Moreover, if $\zeta \in \underline{\operatorname{End}}_{k G}^{m}(F)$ and $\xi \in \underline{\operatorname{End}}_{k G}^{n}(F)$, then $\zeta \circ \xi=\zeta \cdot \xi$.

If $\mathcal{C}$ is the zero subcategory of $k G$-mod so that $F=k$, then this definition produces the usual bimodule structure of $\hat{H}^{*}(G, k)$ on $\widehat{\operatorname{Ext}}_{k G}^{*}(M, N)$. Thus the following proposition generalizes a standard result.

Proposition 3.1. Let $M$ be a $\mathcal{C}$-local $k G$-module, and let $\zeta$ be a homogeneous element of $\operatorname{End}_{k G}^{*}(F)$. Then $\zeta \cdot 1_{M}$ lies in the graded center of $\underline{\operatorname{End}}_{k G}^{*}(M)$, so that $\underline{\operatorname{End}}_{k G}^{*}(M)$ is a graded $\underline{\operatorname{End}}_{k G}^{*}(F)$-algebra.

Proof. The proof is similar to that of Theorem 5.2.3 of [6]. The details are left to the reader.

If $M$ and $N$ are $k G$-modules, we write $\tau: M \otimes N \rightarrow N \otimes M$ for the $k G$ homomorphism that interchanges factors. By abuse of notation the same symbol $\tau$ is used for all such modules $M$ and $N$.

Lemma 3.2. Let $M$ be a $\mathcal{C}$-local $k G$-module. If $\alpha \in \operatorname{End}_{k G}^{m}(F)$ and $n$ is any integer, then

$$
\left(\alpha \cdot 1_{\Omega^{n} k \otimes M}\right)\left(\tau \otimes 1_{M}\right)=1_{\Omega^{n} k} \otimes\left(\alpha \cdot 1_{M}\right) .
$$


Proof. The commutativity of the diagram

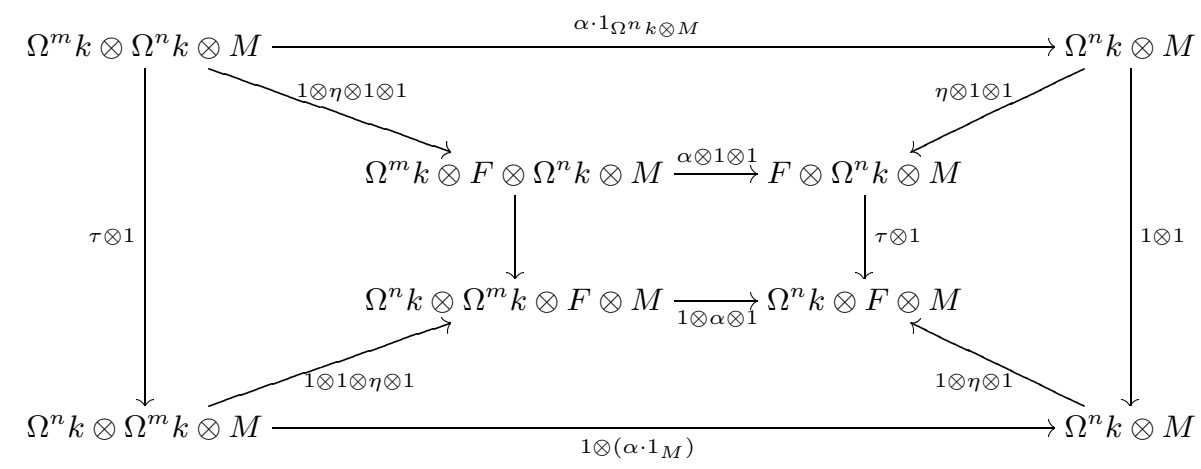

shows that $\alpha \cdot 1_{\Omega^{n} k \otimes M}=\left(1 \otimes\left(\alpha \cdot 1_{M}\right)\right)(\tau \otimes 1)$, as desired.

If $\zeta \in \underline{\operatorname{End}}_{k G}^{n}(F)$, write $F /(\zeta)$ for the module defined by the triangle

$$
\Omega^{n} F \stackrel{\zeta}{\rightarrow} F \stackrel{\lambda}{\rightarrow} F /(\zeta) \stackrel{\mu}{\rightarrow} \Omega^{-1} k \otimes \Omega^{n} F
$$

Note that if $F=k$, then $F /(\zeta)=\Omega^{-1} L_{\zeta}$.

Proposition 3.3. Suppose that $\alpha \in \underline{\operatorname{End}}_{k G}^{m}(F)$ and $\zeta \in \underline{\operatorname{End}}_{k G}^{n}(F)$, and let $M$ be $a \mathcal{C}$-local $k G$-module. Then there is a morphism of triangles of the form

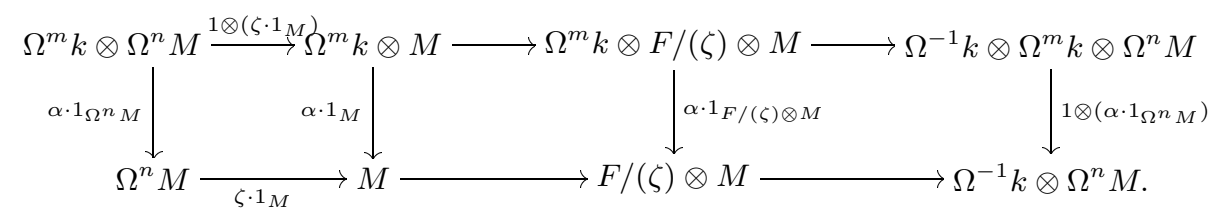

Proof. The top and bottom rows of the commutative diagram

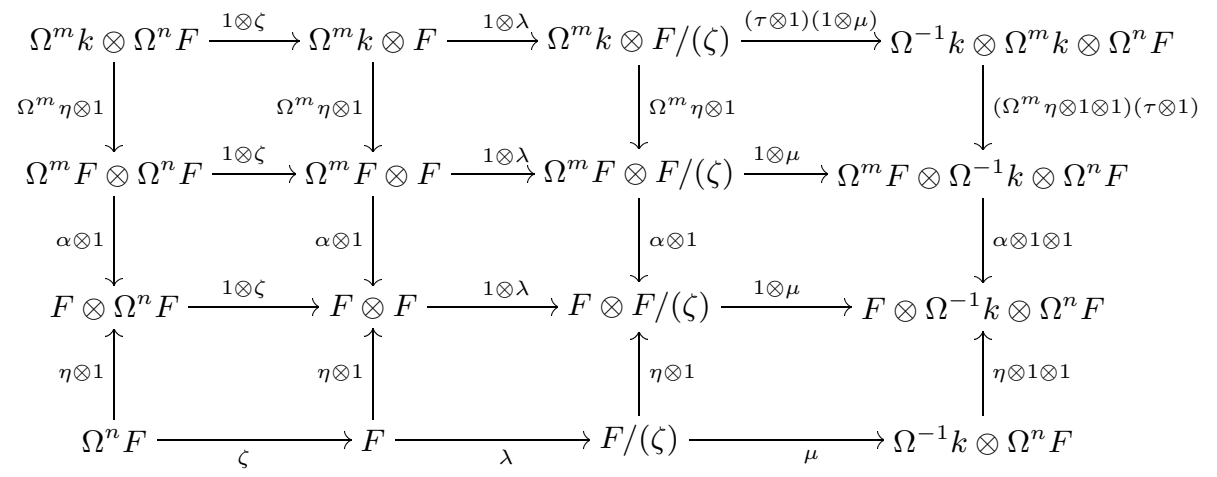

are triangles in $k G$-Mod. Computing the compositions of the maps in the four columns and using the fact that $\left(\alpha \cdot 1_{\Omega^{-1} k \otimes \Omega^{n} F}\right)\left(\tau \otimes 1_{\Omega^{n} F}\right)=1_{\Omega^{-1} k} \otimes\left(\alpha \cdot 1_{\Omega^{n} F}\right)$ 
shows that there is a morphism of triangles

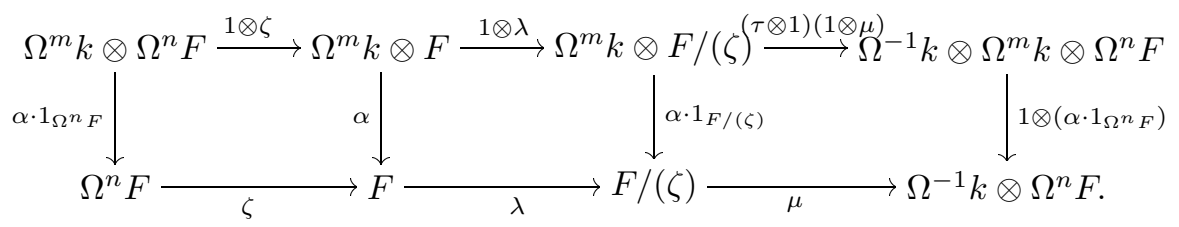

Applying the functor $-\otimes M$ to this diagram and using the stable isomorphism $\eta: M \rightarrow F \otimes M$ now gives the desired result.

Assume that $\zeta_{1}, \ldots, \zeta_{s}$ are homogeneous elements in $\operatorname{End}_{k G}^{*}(F)$, and set $n_{i}=$ $\operatorname{deg} \zeta_{i}$. Define

$$
F /\left(\zeta_{1}, \ldots, \zeta_{s}\right)=F /\left(\zeta_{1}\right) \otimes \cdots \otimes F /\left(\zeta_{s}\right)
$$

For simplicity set $F_{0}=F$ and $F_{i}=F /\left(\zeta_{1}, \ldots, \zeta_{i}\right)$ for $1 \leq i \leq s$. Then $F_{i}$ can also be defined by observing that there is a triangle

$$
\Omega^{n_{i}} F_{i-1} \stackrel{\zeta_{i} \cdot 1_{F_{i-1}}}{\longrightarrow} F_{i-1} \stackrel{\lambda_{i}}{\longrightarrow} F_{i} \stackrel{\mu_{i}}{\longrightarrow} \Omega^{-1} k \otimes \Omega^{n_{i}} F_{i-1} .
$$

Note that the modules $F_{0}, F_{1}, \ldots, F_{s}$ are $\mathcal{C}$-local by induction.

Proposition 3.4. Let $\zeta_{1}, \ldots, \zeta_{s}$ be a regular sequence in $\operatorname{End}_{k G}^{*}(F)$. Then for $1 \leq i \leq s$ the elements $\zeta_{i} \cdot 1, \ldots, \zeta_{s} \cdot 1$ form a regular sequence in $\operatorname{End}_{k G}^{*}\left(F_{i-1}\right)$, and

$$
\underline{\operatorname{Hom}}_{k G}^{*}\left(F_{i-1}, F_{i}\right) \cong \underline{\operatorname{End}}_{k G}^{*}\left(F_{i-1}\right) /\left(\zeta_{i} \cdot 1\right)
$$

as graded $\underline{\operatorname{End}}_{k G}^{*}(F)$-bimodules. Moreover, the composition

$$
\underline{\operatorname{End}}_{k G}^{*}\left(F_{i}\right) \stackrel{\lambda_{i}^{*}}{\longrightarrow} \underline{\operatorname{Hom}}_{k G}^{*}\left(F_{i-1}, F_{i}\right) \cong \underline{\operatorname{End}}_{k G}^{*}\left(F_{i-1}\right) /\left(\zeta_{i} \cdot 1\right)
$$

is an epimorphism of rings.

Proof. Assume by induction that $i \geq 1$ and that $\zeta_{i} \cdot 1, \ldots, \zeta_{s} \cdot 1$ is a regular sequence in $\underline{\operatorname{End}}_{k G}^{*}\left(F_{i-1}\right)$. Applying the functor $\underline{\operatorname{Hom}}_{k G}^{n}\left(F_{i-1},-\right)$ to the triangle (3.1) gives a short exact sequence

$$
0 \rightarrow \underline{\operatorname{End}}_{k G}^{n-n_{i}}\left(F_{i-1}\right) \stackrel{\left(\zeta_{i} \cdot 1\right)_{*}}{\longrightarrow} \underline{\operatorname{End}}_{k G}^{n}\left(F_{i-1}\right) \stackrel{\lambda_{i *}}{\longrightarrow} \underline{\operatorname{Hom}}_{k G}^{n}\left(F_{i-1}, F_{i}\right) \rightarrow 0
$$

because $\zeta_{i} \cdot 1$ is a regular element of $\operatorname{End}_{k G}^{*}\left(F_{i-1}\right)$. It follows that there is an isomorphism

$$
\underline{\operatorname{Hom}}_{k G}^{*}\left(F_{i-1}, F_{i}\right) \cong \underline{\operatorname{End}}_{k G}^{*}\left(F_{i-1}\right) /\left(\zeta_{i} \cdot 1\right)
$$

of graded right $\operatorname{End}_{k G}^{*}(F)$-modules. Proposition 3.3 implies that this is actually an isomorphism of graded $\underline{\operatorname{End}}_{k G}^{*}(F)$-bimodules. For example, if $\alpha \in \underline{\operatorname{End}}_{k G}^{m}(F)$ and $f \in \underline{\operatorname{End}}_{k G}^{n}\left(F_{i-1}\right)$, then

$\lambda_{i *}(\alpha \cdot f)=\lambda_{i} \circ\left(\alpha \cdot 1_{F_{i-1}}\right) \circ\left(1_{\Omega^{m} k} \otimes f\right)=\left(\alpha \cdot 1_{F_{i}}\right) \circ\left(1_{\Omega^{m} k} \otimes \lambda_{i *}(f)\right)=\alpha \cdot \lambda_{i *}(f)$.

Applying the functor $\underline{\operatorname{Hom}}_{k G}^{n}\left(-, F_{i}\right)$ to the triangle (3.1) gives a long exact sequence

$$
\cdots \rightarrow \underline{\operatorname{End}}_{k G}^{n}\left(F_{i}\right) \stackrel{\lambda_{i}^{*}}{\longrightarrow} \underline{\operatorname{Hom}}_{k G}^{n}\left(F_{i-1}, F_{i}\right) \stackrel{\left(\zeta_{i} \cdot 1\right)^{*}}{\longrightarrow} \underline{\operatorname{Hom}}_{k G}^{n+n_{i}}\left(F_{i-1}, F_{i}\right) \rightarrow \cdots .
$$

Because $\underline{\operatorname{Hom}}_{k G}^{*}\left(F_{i-1}, F_{i}\right) \cong \underline{\operatorname{End}}_{k G}^{*}\left(F_{i-1}\right) /\left(\zeta_{i} \cdot 1\right)$, it follows that there is a short exact sequence

$$
0 \rightarrow \underline{\operatorname{Hom}}_{k G}^{*}\left(F_{i-1}, F_{i}\right) \rightarrow \underline{\operatorname{End}}_{k G}^{*}\left(F_{i}\right) \stackrel{\lambda_{i}^{*}}{\longrightarrow} \underline{\operatorname{Hom}}_{k G}^{*}\left(F_{i-1}, F_{i}\right) \rightarrow 0
$$


in which the first map has degree $1-n_{i}$. Moreover, the maps in this sequence are homomorphisms of graded $\operatorname{End}_{k G}^{*}(F)$-bimodules by Proposition 3.3. The elements $\zeta_{i} \cdot 1, \ldots, \zeta_{s} \cdot 1$ form a regular sequence in $\operatorname{End}_{k G}^{*}\left(F_{i-1}\right)$, so $\zeta_{i+1}, \ldots, \zeta_{s}$ is a regular sequence on the $\underline{\operatorname{End}}_{k G}^{*}(F)$-bimodule $\underline{\operatorname{End}}_{k G}^{*}\left(F_{i-1}\right) /\left(\zeta_{i} \cdot 1\right) \cong \underline{\operatorname{Hom}}_{k G}^{*}\left(F_{i-1}, F_{i}\right)$ and hence also on $\underline{\operatorname{End}}_{k G}^{*}\left(F_{i}\right)$. Finally, it is easy to check that the composition

$$
\underline{\operatorname{End}}_{k G}^{*}\left(F_{i}\right) \stackrel{\lambda_{i}^{*}}{\longrightarrow} \underline{\operatorname{Hom}}_{k G}^{*}\left(F_{i-1}, F_{i}\right) \cong \underline{\operatorname{End}}_{k G}^{*}\left(F_{i-1}\right) /\left(\zeta_{i} \cdot 1\right)
$$

is an epimorphism of rings. This completes the proof.

Theorem 3.5. Let $\zeta_{1}, \ldots, \zeta_{s}$ be a regular sequence in $\operatorname{End}_{k G}^{*}(F)$. Then there is an epimorphism

$$
\underline{\operatorname{End}}_{k G}^{*}\left(F /\left(\zeta_{1}, \ldots, \zeta_{s}\right)\right) \rightarrow \underline{\operatorname{End}}_{k G}^{*}(F) /\left(\zeta_{1}, \ldots, \zeta_{s}\right)
$$

whose kernel is an ideal I satisfying $I^{2^{s}}=0$.

Proof. For $1 \leq i \leq s$ let $n_{i}=\operatorname{deg} \zeta_{i}$, and let $\phi_{i}: \operatorname{End}_{k G}^{*}\left(F_{i}\right) \rightarrow \operatorname{End}_{k G}^{*}\left(F_{i-1}\right) /\left(\zeta_{i} \cdot 1\right)$ be the epimorphism given by Proposition 3.4. If $\zeta \in \operatorname{End}_{k G}^{*}(F)$ is homogeneous, then $\phi_{i}$ sends $\zeta \cdot 1_{F_{i}}$ to $\zeta \cdot 1_{F_{i-1}}+\left(\zeta_{i} \cdot 1\right)$ by Proposition 3.3. Thus $\phi_{i}$ induces an epimorphism

$$
\theta_{i}: \underline{\operatorname{End}}_{k G}^{*}\left(F_{i}\right) /\left(\zeta_{i+1} \cdot 1, \ldots, \zeta_{s} \cdot 1\right) \rightarrow \underline{\operatorname{End}}_{k G}^{*}\left(F_{i-1}\right) /\left(\zeta_{i} \cdot 1, \ldots, \zeta_{s} \cdot 1\right),
$$

and the composition $\theta=\theta_{1} \circ \cdots \circ \theta_{s} \operatorname{maps}_{\operatorname{End}_{k G}^{*}}^{*}\left(F_{s}\right)$ onto $\underline{\operatorname{End}}_{k G}^{*}(F) /\left(\zeta_{1}, \ldots, \zeta_{s}\right)$.

It remains to show that $I=\operatorname{Ker} \theta$ satisfies $I^{2^{s}}=0$. We will prove this result by showing that $\operatorname{Ker} \theta_{i}$ is an ideal of square zero for $1 \leq i \leq s$. Consider the triangle

$$
\begin{aligned}
\Omega^{n} k \otimes \Omega^{n_{i}} F_{i-1} & \stackrel{1 \otimes\left(\zeta_{i} \cdot 1\right)}{\longrightarrow} \Omega^{n} k \otimes F_{i-1} \stackrel{1 \otimes \lambda_{i}}{\longrightarrow} \Omega^{n} k \otimes F_{i} \\
\stackrel{(\tau \otimes 1)\left(1 \otimes \mu_{i}\right)}{\longrightarrow} & \Omega^{-1} k \otimes \Omega^{n} k \otimes \Omega^{n_{i}} F_{i-1} .
\end{aligned}
$$

If $\beta \in \underline{\operatorname{End}}_{k G}^{n}\left(F_{i}\right)$ satisfies $0=\lambda_{i}^{*}(\beta)=\beta\left(1_{\Omega^{n} k} \otimes \lambda_{i}\right)$, then there is a map $\beta^{\prime}$ :

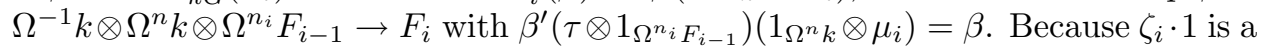
regular element of $\operatorname{End}_{k G}^{*}\left(F_{i-1}\right)$ and $\mu_{i} \beta^{\prime}: \Omega^{-1} k \otimes \Omega^{n} k \otimes \Omega^{n_{i}} F_{i-1} \rightarrow \Omega^{-1} k \otimes \Omega^{n_{i}} F_{i-1}$ satisfies

$$
\left(1_{\Omega^{-1} k} \otimes\left(\zeta_{i} \cdot 1\right)\right)\left(\mu_{i} \beta^{\prime}\right)=0,
$$

it follows that $\mu_{i} \beta^{\prime}=0$. This fact implies that $\operatorname{Ker} \lambda_{i}^{*}=\operatorname{Ker} \phi_{i}$ has square zero. Because the vertical maps in the commutative diagram

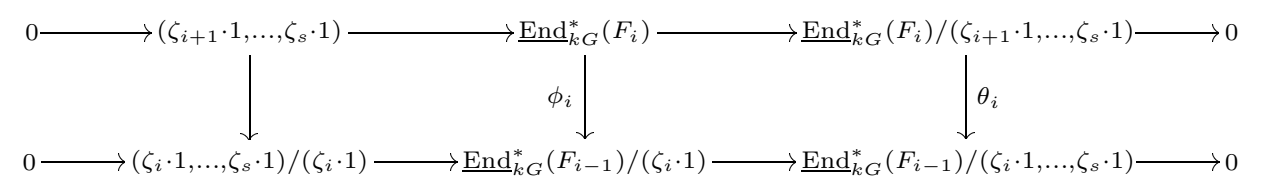

are all epimorphisms, it follows that $\operatorname{Ker} \theta_{i}$ is an ideal of square zero for all $i$. This completes the proof.

\section{FAilure of CANCELlation}

In this section we apply the previous results to construct a class of examples of modules for which the cancellation property fails. In particular, when $p$ is an odd prime, we exhibit countably generated modules $M$ and $N$ for the group $(\mathbb{Z} / p)^{7}$ such that

$$
M \oplus M \oplus M \cong M \oplus N
$$


but $M \oplus M \neq N$. When $p=2$, we give a similar example in which the cancellation property fails for $(\mathbb{Z} / 2)^{9}$. The idea of the construction is to begin by producing a module such that the cancellation property fails for finitely generated projective modules over its endomorphism ring. This step is based upon some related examples of Raynaud [12], and we begin by describing these examples.

Let $k$ be an algebraically closed field of characteristic $p>0$. Let $q$ and $n$ be integers with $1<q<n$, and let $\delta_{j l}$ denote the Kronecker delta function for $1 \leq j, l \leq n-q$. Set

$$
\begin{aligned}
& R=k\left[X_{j i}, Y_{i j}\right]_{1 \leq i \leq n, 1 \leq j \leq n-q}, \\
& E=R / \mathfrak{A},
\end{aligned}
$$

where $\mathfrak{A}$ is the ideal generated by the elements ${ }^{1}$

$$
\left(\sum_{i=1}^{n} X_{j i} Y_{i l}\right)-\delta_{j l}
$$

for $1 \leq j, l \leq n-q$. We use the same symbols $X_{j i}$ and $Y_{i j}$ to denote the images in $E$ of $X_{j i}, Y_{i j} \in R$. Let $X$ denote the $(n-q) \times n$ matrix $\left(X_{j i}\right)$ with entries in $E$, and let $Y$ denote the $n \times(n-q)$ matrix $\left(Y_{i j}\right)$. Then the relations (4.1) show that the product $X Y$ is the $(n-q) \times(n-q)$ identity matrix, so $e=Y X$ satisfies $e^{2}=e$. If $e$ is regarded as an idempotent endomorphism of a free module $E^{n}$ of rank $n$, then

$$
E^{n}=e E^{n} \oplus(1-e) E^{n} .
$$

Regarding $X$ and $Y$ as homomorphisms $X: E^{n} \rightarrow E^{n-q}$ and $Y: E^{n-q} \rightarrow E^{n}$ of right $E$-modules, we see that

$$
e E^{n} \cong E^{n-q},
$$

so that $(1-e) E^{n}$ is a finitely generated projective $E$-module that is stably free of rank q. In Corollaire 6.3 of [12] Raynaud uses Steenrod operations in étale cohomology to show that the modules $(1-e) E^{n}$ are not free, except possibly when $p=q=2$ or when $p=q=3$.

Let $G$ be an elementary abelian $p$-group of rank $2 n+1$. If $p=2$, then $H^{*}(G, k)$ is a polynomial ring $k\left[\zeta_{1}, \ldots, \zeta_{2 n+1}\right]$ in $2 n+1$ generators of degree one. If $p$ is odd, then $H^{*}(G, k)$ is the tensor product of a polynomial ring $k\left[\zeta_{1}, \ldots, \zeta_{2 n+1}\right]$ and an exterior algebra $\Lambda\left(\eta_{1}, \ldots, \eta_{2 n+1}\right)$; the elements $\zeta_{1}, \ldots, \zeta_{2 n+1}$ have degree two, and the elements $\eta_{1}, \ldots, \eta_{2 n+1}$ have degree one. Let $\mathcal{C}$ be the subcategory of $k G$-mod consisting of all finitely generated modules $M$ such that $V_{G}(M) \subseteq V_{G}\left(\zeta_{2 n+1}\right)$, and set $F=F_{\mathcal{C}}(k)$. Then $\underline{\operatorname{End}}_{k G}^{*}(F)$ is isomorphic to $\hat{H}^{*}(G, k)\left[\zeta_{2 n+1}^{-1}\right]$. Set $\alpha_{i}=\zeta_{2 n+1}^{-1} \zeta_{i}$ for $1 \leq i \leq 2 n$. We claim that every nonzero element of $k\left[\alpha_{1}, \ldots, \alpha_{2 n}\right]$ is regular in $\underline{\operatorname{End}}_{k G}^{*}(F)$. Indeed, Theorem 3.3 of [2] shows that the product of any two elements of negative degree is zero in $\hat{H}^{*}(G, k)$. Lemma 2.1 of [2] then implies that if $\xi \in$ $\hat{H}^{-d}(G, k)$ for some $d>0$, then $\zeta_{2 n+1}^{d} \xi=0$, so that $\xi=0$ in $\hat{H}^{*}(G, k)\left[\zeta_{2 n+1}^{-1}\right]$. Thus

$$
\underline{\operatorname{End}}_{k G}^{*}(F)=\hat{H}^{*}(G, k)\left[\zeta_{2 n+1}^{-1}\right]=H^{*}(G, k)\left[\zeta_{2 n+1}^{-1}\right],
$$

and it follows that every nonzero element of $k\left[\alpha_{1}, \ldots, \alpha_{2 n}\right]$ is regular in $\operatorname{End}_{k G}^{*}(F)$.

If $q=n-1$, then the relations (4.1) reduce to a single equation in $E$ of the form $\sum_{i=1}^{n} X_{1 i} Y_{i 1}-1=0$. Let $\phi: k\left[\alpha_{1}, \ldots, \alpha_{2 n}\right] \rightarrow E$ be the ring epimorphism

\footnotetext{
${ }^{1}$ There is a misprint in [12] at this point: the term $-\delta_{j l}$ is omitted.
} 
satisfying $\phi\left(\alpha_{i}\right)=X_{1 i}$ and $\phi\left(\alpha_{n+i}\right)=Y_{i 1}$ for $1 \leq i \leq n$. Then

$$
\xi=\sum_{i=1}^{n} \alpha_{i} \alpha_{n+i}-1
$$

is in $\operatorname{Ker} \phi$, and $\phi$ induces an isomorphism of $\operatorname{End}_{k G}(F) /(\xi)$ modulo its nilradical onto $E$. Because $\xi$ is regular in $\operatorname{End}_{k G}^{*}(F)$, Theorem 3.5 implies that if $\mathfrak{N}_{0}$ is the nilradical of $E_{0}=\operatorname{End}_{k G}(F /(\xi))$, then $E_{0} / \mathfrak{N}_{0} \cong E$. We use this isomorphism to identify $E_{0} / \mathfrak{N}_{0}$ with $E$.

Let $X^{\prime}$ and $Y^{\prime}$ be matrices with entries in $E_{0}$ such that the reduction modulo $\mathfrak{N}_{0}$ of $X^{\prime}$ is $X$ and of $Y^{\prime}$ is $Y$. Then $X^{\prime} Y^{\prime}-1 \in \mathfrak{N}_{0}$, so there is an integer $t>0$ such that $\left(X^{\prime} Y^{\prime}-1\right)^{t}=0$. Set $X_{0}=X^{\prime}$ and

$$
Y_{0}=Y^{\prime} \sum_{i=1}^{t}(-1)^{i-1}\left(\begin{array}{l}
t \\
i
\end{array}\right)\left(X^{\prime} Y^{\prime}\right)^{i-1} .
$$

Then $X_{0}$ and $Y_{0}$ are matrices with entries in $E_{0}$ such that $X_{0} Y_{0}=1$ and the reduction modulo $\mathfrak{N}_{0}$ of $X_{0}$ is $X$ and of $Y_{0}$ is $Y$. In particular, $e_{0}=Y_{0} X_{0}$ is an idempotent endomorphism of $E_{0}^{n}$ lifting the idempotent $e$. Thus $E_{0}$ satisfies

$$
E_{0}^{n}=e_{0} E_{0}^{n} \oplus\left(1-e_{0}\right) E_{0}^{n}
$$

and $e_{0} E_{0}^{n} \cong E_{0}$. Moreover, if $(1-e) E^{n}$ is not a free $E$-module, then $\left(1-e_{0}\right) E_{0}^{n}$ is not a free $E_{0}$-module.

The following proposition provides the connection between the above discussion and modules over a group algebra.

Proposition 4.1. Let $G$ be a finite group, and let $M$ be a $k G$-module with endomorphism ring $E_{0}$. Let $e_{0}$ and $f_{0}$ be idempotent elements of $\operatorname{Mat}_{n}\left(E_{0}\right)$. Then $e_{0} E_{0}^{n} \cong f_{0} E_{0}^{n}$ as right $E_{0}$-modules if and only if $e_{0} M^{n} \cong f_{0} M^{n}$ as left $k G$-modules.

Proof. Observe that there are isomorphisms

$$
\operatorname{Hom}_{E_{0}}\left(e_{0} E_{0}^{n}, f_{0} E_{0}^{n}\right) \cong f_{0} \operatorname{Mat}_{n}\left(E_{0}\right) e_{0} \cong \operatorname{Hom}_{k G}\left(e_{0} M^{n}, f_{0} M^{n}\right)
$$

and

$$
\operatorname{Hom}_{E_{0}}\left(f_{0} E_{0}^{n}, e_{0} E_{0}^{n}\right) \cong e_{0} \operatorname{Mat}_{n}\left(E_{0}\right) f_{0} \cong \operatorname{Hom}_{k G}\left(f_{0} M^{n}, e_{0} M^{n}\right) .
$$

Thus $e_{0} E_{0}^{n} \cong f_{0} E_{0}^{n}$ if and only if there are matrices $A \in f_{0} \operatorname{Mat}_{n}\left(E_{0}\right) e_{0}$ and $B \in$ $e_{0} \operatorname{Mat}_{n}\left(E_{0}\right) f_{0}$ with $A B=f_{0}$ and $B A=e_{0}$, which occurs precisely when $e_{0} M^{n} \cong$ $f_{0} M^{n}$.

Proposition 4.1 implies that there is an isomorphism

$$
(F /(\xi)) \oplus(F /(\xi))^{n-1}=(F /(\xi))^{n} \cong(F /(\xi)) \oplus\left(1-e_{0}\right)(F /(\xi))^{n},
$$

and $\left(1-e_{0}\right)(F /(\xi))^{n}$ is not isomorphic to $(F /(\xi))^{n-1}$ as long as $(1-e) E^{n}$ is not a free $E$-module. For $p=2$ Raynaud's result implies, therefore, that $\left(1-e_{0}\right)(F /(\xi))^{n}$ is not isomorphic to $(F /(\xi))^{n-1}$ when $n=4$ and $G=(\mathbb{Z} / 2)^{9}$; similarly, for $p>2$ these modules are not isomorphic when $n=3$ and $G=(\mathbb{Z} / p)^{7}$. Thus (4.2) shows that the cancellation property fails in these cases. Brenner and Ringel [7] show by more abstract reasoning that the cancellation property fails as long as the $p$-rank of $G$ is at least two.

More generally, if $1<q<n$ and $G$ is an elementary abelian $p$-group of rank $2 n(n-q)+1$, then Raynaud's examples show that the cancellation property fails for a module of the form $F /\left(\xi_{1}, \ldots, \xi_{(n-q)^{2}}\right)$. The details are left to the reader. 


\section{REFERENCES}

[1] D. J. Benson, Cohomology of modules in the principal block of a finite group, New York J. Math. 1 (1995), 196-205. MR 96h:20095

[2] D. J. Benson and J. F. Carlson, Products in negative cohomology, J. Pure Appl. Algebra 82 (1992), 107-129. MR 93i:20058

[3] D. J. Benson, J. F. Carlson, and J. Rickard, Complexity and varieties for infinitely generated modules, I, Math. Proc. Cambridge Phil. Soc. 118 (1995), 223-243. MR 96j:20006

[4] D. J. Benson, J. F. Carlson, and J. Rickard, Complexity and varieties for infinitely generated modules, II, Math. Proc. Cambridge Phil. Soc. 120 (1996), 597-615. MR 97f:20008

[5] D. J. Benson, J. F. Carlson, and J. Rickard, Thick subcategories of the stable module category, Fundamenta Mathematicae 153 (1997), 59-80. MR 98g:20021

[6] D. J. Benson and G. Ph. Gnacadja, Phantom maps and purity in modular representation theory, to appear in Fundamenta Mathematicae.

[7] S. Brenner and C. Ringel, Pathological modules over tame rings, J. London Math. Soc. (2) 14 (1976), 207-215. MR 55:5697

[8] J. F. Carlson, P. W. Donovan, and W. W. Wheeler, Complexity and quotient categories for group algebras, J. Pure Appl. Algebra 93 (1994), 147-167. MR 95a:20009

[9] J. F. Carlson and W. W. Wheeler, Homomorphisms in higher complexity quotient categories, Seattle Conference on Representations of Finite Groups, Proc. Sympos. Pure Math., vol. 63, Amer. Math. Soc., Providence, RI, 1998, pp. 115-155. CMP 98:08

[10] J. A. Green, On the indecomposable representations of a finite group, Math. Z. 70 (1959), 430-445. MR 24:A1304

[11] D. Happel, Triangulated Categories in the Representation Theory of Finite Dimensional Algebras, Cambridge Univ. Press, Cambridge, 1998. MR 89e:16035

[12] M. Raynaud, Modules projectifs universels, Invent. Math. 6 (1968), 1-26. MR 38:4462

[13] J. Rickard, Idempotent modules in the stable category, J. London Math. Soc. (2) 56 (1997), 149-170. MR 98d:20058

Department of Mathematics, University of Georgia, Athens, Georgia 30602

E-mail address: djb@byrd.math.uga.edu

Current address, Wayne Wheeler: Department of Mathematics and Computer Science, University of Leicester, University Road, Leicester LE1 7RH, England

E-mail address: www@sloth.math.uga.edu 